\title{
Discrete Neuroanatomical Networks Are Associated with Specific Cognitive Abilities in Old Age
}

\author{
Wei Wen, ${ }^{1,2}$ Wanlin Zhu, ${ }^{1,2}$ Yong He, ${ }^{4}$ Nicole A. Kochan, ${ }^{1,2}$ Simone Reppermund, ${ }^{1}$ Melissa J. Slavin, ${ }^{1}$ Henry Brodaty, ${ }^{1,3}$ \\ John Crawford, ${ }^{1,2}$ Aihua Xia, ${ }^{5}$ and Perminder Sachdev ${ }^{1,2}$ \\ ${ }^{1}$ School of Psychiatry, University of New South Wales, Sydney, New South Wales 2052, Australia, ${ }^{2}$ Neuropsychiatric Institute and ${ }^{3}$ Academic Department \\ for Old Age Psychiatry, Prince of Wales Hospital, Randwick, New South Wales 2031, Australia, ${ }^{4}$ State Key Laboratory of Cognitive Neuroscience and \\ Learning, Beijing Normal University, Beijing 100875, China, and ${ }^{5}$ Department of Mathematics and Statistics, University of Melbourne, Melbourne, Victoria \\ 3010, Australia
}

There have been many attempts at explaining age-related cognitive decline on the basis of regional brain changes, with the usual but inconsistent findings being that smaller gray matter volumes in certain brain regions predict worse cognitive performance in specific domains. Additionally, compromised white matter integrity, as suggested by white matter hyperintensities or decreased regional white matter fractional anisotropy, has an adverse impact on cognitive functions. The human brain is, however, a network and it may be more appropriate to relate cognitive functions to properties of the network rather than specific brain regions. We report on graph theory-based analyses of diffusion tensor imaging tract-derived connectivity in a sample of 342 healthy individuals aged 72-92 years. The cognitive domains included processing speed, memory, language, visuospatial, and executive functions. We examined the association of these cognitive assessments with both the connectivity of the whole brain network and individual cortical regions. We found that the efficiency of the whole brain network of cortical fiber connections had an influence on processing speed and visuospatial and executive functions. Correlations between connectivity of specific regions and cognitive assessments were also observed, e.g., stronger connectivity in regions such as superior frontal gyrus and posterior cingulate cortex were associated with better executive function. Similar to the relationship between regional connectivity efficiency and age, greater processing speed was significantly correlated with better connectivity of nearly all the cortical regions. For the first time, regional anatomical connectivity maps related to processing speed and visuospatial and executive functions in the elderly are identified.

\section{Introduction}

Decline in information-processing resources, such as working memory capacity, executive function, attention regulation, and processing speed, is likely to occur in aging due to structural brain changes (Buckner, 2004; Raz et al., 2005). There have been many studies relating brain structure to cognitive function, with the majority being focused on regional structural changes at the macroanatomical level. Worse cognitive performances in specific domains are often found to be associated with smaller gray matter volumes in certain regions (Salat et al., 2002; Driscoll et al., 2003). White matter hyperintensity (WMH) (Fazekas et al., 1993; Gunning-Dixon and Raz, 2003) and decreased regional white matter fractional anisotropy (FA) (Kennedy and Raz, 2009) adversely impact cognitive functions. This is possibly due to the continuing enthusiasm of researchers for the traditional localiza-

\footnotetext{
Received Aug. 5, 2010; revised Oct. 22, 2010; accepted Nov. 16, 2010.

This research was supported by the National Health and Medical Research Council (NHMRC Program Grant ID 350833, NHMRC Project Grant ID 510175), the Australian Research Council (ARC DP-0774213), the Natural Science Foundation of China (NSFC 30870667), and the International Research Collaboration Scheme of the University of New South Wales. We thank the study participants and interviewers. We also thank Kate Crosbie and Angie Russell for their assistance in the manuscript preparation.

Correspondence should be addressed to Wei Wen, School of Psychiatry, University of New South Wales, Sydney, NSW 2052, Australia. E-mail:w.wen@unsw.edu.au.

DOI:10.1523/JNEUROSCI.4085-10.2011

Copyright $\odot 2011$ the authors $\quad 0270-6474 / 11 / 311204-09 \$ 15.00 / 0$
}

tionist view of the brain and the increasing accessibility of brainmapping tools to the researchers.

While particular brain regions are important for specific functions, the capacity of information flow within and between regions is also crucial. For instance, besides the atrophy often seen in the medial temporal lobe, parietal lobe, and posterior cingulate and prefrontal cortex, Alzheimer's disease $(\mathrm{AD})$ is a progressive impairment of fiber-track connectivity characterized by the loss of afferent and efferent connections of regional neocortical areas associated with the death of pyramidal neurons (Morrison and Hof, 2002). Cerebral small-vessel disease, manifesting as lacunar infarcts (Chen et al., 2009) and WMH, has been found to slow down information processing and lead to decline in executive functions (Jorm et al., 2004; Wen and Sachdev, 2004; Prins et al., 2005). It is therefore important to examine the characteristics of the brain network.

While the large-scale connectivity structure of the human brain represents a relatively invariant characteristic (Sporns et al., 2005), the precise combinations and topological patterns may be influenced by many factors, such as genetic differences, sex, aging, and/or pathologies. These factors may determine specific constraints on brain dynamics, and thus contribute to the variabilities of brain networks. An increasing number of brain network studies have emerged recently using graph theoretical analysis of structural and functional systems (Bullmore and 
Sporns, 2009). Recently, in vivo human anatomical networks have been constructed using diffusion tensor imaging (DTI) (Gong et al., 2009a) and diffusion spectrum imaging data (Hagmann et al., 2008). However, apart from one recent DTI network study on 79 healthy young adults reporting that network efficiency is associated with intelligence (Li et al., 2009), no other study has related DTI network properties to cognitive functions.

In this work, we constructed corticocortical networks in a cohort of older community-dwelling, nondemented native English speakers aged between $72-92$ years using DTI tractography. We examined correlations of brain cortical connectivity with cognitive performances in a range of cognitive domains. We hypothesized that some cognitive processes will be more dependent on effective information transfer and integration between connected clusters of particular cortical regions, while others will be more globally distributed; thus, both the global and local cortical networks will differentially influence cognitive performance in different domains.

\section{Materials and Methods \\ Subjects}

Participants were drawn from Wave 2 of the Sydney Memory and Aging Study, a longitudinal study examining the predictors of cognitive decline in an elderly, nondemented, community-dwelling sample. They were recruited randomly through the electoral roll from two electorates of Eastern Sydney, Australia, where registration on the electoral roll is compulsory. Participants were excluded from the study if any of the following was evident: dementia, mental retardation, psychotic disorder, including schizophrenia or bipolar disorder, multiple sclerosis, motor neuron disease, active malignancy, or English inadequate to complete a basic assessment. At study entry, participants consisted of 1037 individuals aged 70-90 years, of whom 542 (52.3\%) had an MRI scan at Wave 1. Those who had a Wave 1 MRI scan were offered a 2 year follow-up MRI at Wave 2. A total of 424 subjects had Wave 2 scans. After removing 19 scans with artifacts (DTI artifacts: 9; T1-weighted artifacts: 10-we used T1weighted scans to obtain brain parcellations, etc.), 405 were considered usable and were of high quality. Finally, 342 (male/female = 158/184; mean age $=79.70 \pm 4.55$ ) of these 405 subjects were entered into the study after exclusion of 63 participants on the basis of having nonEnglish-speaking background (acquired conversational English at $\geq 10$ years of age), incomplete information, or extreme outliers on the neuropsychological test scores ( $>3$ interquartile lengths below/above first/ third quartile). The study was approved by the Ethics Committee of the University of New South Wales.

\section{MRI acquisition}

All subjects were scanned using a Philips 3T Achieva Quasar Dual scanner (Philips Medical Systems) located at the Prince of Wales Medical Research Institute, Sydney. Foam pads and headphones were used to reduce head motion and scanner noise. To increase the signal-to-noise ratio (SNR), all of the subjects were scanned twice for both DTI and 3D T1-weighted sequences.

Diffusion tensor imaging scans. A single-shot echo-planar imaging (EPI) sequence $(\mathrm{TR}=7115 \mathrm{~ms}, \mathrm{TE}=70 \mathrm{~ms}$ ) was used. Diffusion sensitizing gradients were applied along 32 non-collinear directions $(b=1000$ $\left.\mathrm{s} / \mathrm{mm}^{2}\right)$, together with a non-diffusion-weighted acquisition $(b=0$ $\mathrm{s} / \mathrm{mm}^{2}$ ). For each DTI scan, 55 axial slices were collected. The field of view was $240 \mathrm{~mm} \times 240 \mathrm{~mm} \times 137.5 \mathrm{~mm}$ with acquisition matrix $96 \times$ 96 and zero filled into $240 \times 240$; and slice thickness $2.5 \mathrm{~mm}$ with no gap, yielding $1 \mathrm{~mm} \times 1 \mathrm{~mm} \times 2.5$ voxels. Two extra non-diffusion-weighted $\left(b=0 \mathrm{~s} / \mathrm{mm}^{2}\right)$ EPIs were separately acquired and then combined with DTI scans for higher SNR.

3D T1-weighted structural scans. The main parameters for 3D T1weighted structural MRI were as follows: $\mathrm{TR}=6.39 \mathrm{~ms}$, $\mathrm{TE}=2.9 \mathrm{~ms}$, flip angle $=8^{\circ}$, matrix size $=256 \times 256$, FOV $=256 \times 256 \times 190$, and slice thickness = $1 \mathrm{~mm}$ with no gap between; yielding $1 \mathrm{~mm} \times 1 \mathrm{~mm} \times 1 \mathrm{~mm}$ isotropic voxels. Both the DTI data and T1-weighted data were visually inspected for apparent artifacts arising from subject head motion and instrument malfunction. Those showing apparent artifacts were removed from the study.

\section{Neuropsychological testing battery and cognitive domains}

A comprehensive battery of neuropsychological tests, covering five cognitive domains of processing speed and memory, language, visuospatial, and executive function, was administered by trained psychologists. The processing speed domain was defined by two tests, Digit Symbol-Coding (Wechsler, 1997) and Trail Making Part A (Reitan and Wolfson, 1985; Strauss et al., 2006). The spatial domain was defined by a single test, Block Design (Wechsler, 1981). The executive domain was defined by the Stroop interference score (MacLeod, 1991) (difference between incongruent color-word naming and color naming), a phonemic fluency test (FAS), and Trail Making Test Part B (Strauss et al., 2006). The memory domain comprised Logical Memory Story A (immediate and delayed recall) (Wechsler, 1997), the Rey Auditory Verbal Learning Test (shortterm and long-term delayed recall, and learning score; sum of five learning trials) (Rey, 1964), and the Benton Visual Retention test (Benton and Spreen, 1996). The language domain score was obtained from the Boston Naming Test (30 items) (Kaplan et al., 2001) and a semantic fluency test (Animals) (Spreen and Benton, 1969). For each participant, we calculated the $Z$-scores of the individual tests using the sample means and SDs. A score for each domain was then calculated as the average of the $Z$-scores of tests comprising the domain. Finally, to simplify the interpretation of results, each of these scores was converted to a $Z$-score to produce standardized scores for each of the five cognitive domains that were included in the analyses, i.e., processing speed, spatial, executive, language, and memory.

\section{Construction of structural networks}

One structural network graph was generated for each subject using the subject's DTI data. In graph theory, a network is defined as a set of nodes and the edges between them (Bullmore and Sporns, 2009). Due to the inability of DTI tractography to differentiate efferent from afferent fibers, the graphs we constructed were all undirected. We describe here some of the major steps that we went through from image preprocessing to computing of the nodes and edges of the graph.

Preprocessing of DTI. The software package FSL version 4.1.2. (FMRIB Software Library) (Smith et al., 2004) (http://www.fmrib.ox.ac.uk/fsl) was used for most of the DTI preprocessing steps. The two DTI scans acquired for each subject for an increased SNR were concatenated to form a single combined set. Distortions in the diffusion tensor images caused by eddy currents and head motions were corrected using the first scan's b0 image as the target. The eddy current- and motion-corrected combined image was then split into two sets of images, which were averaged. The average diffusion image was used to calculate the threedimensional maps of the diffusion tensor and the FA.

Definition and computation of network nodes. Regional cortical areas were defined as the network nodes, with only cortical, and not subcortical, regions being used for reasons of accuracy. While satisfactory gray and white matter segmentation could be achieved for the cortex, accurately differentiating subcortical boundaries proved to be difficult. The software package FreeSurfer (http://surfer.nmr.mgh.harvard.edu/) version 4.5.0 was used for coregistering and averaging two sets of T1weighted images of each subject and then performing tissue segmentation to obtain the white matter and cortex boundary. The labeled mesh that defined 68 gyral-based cortical regions (34 on each hemisphere) on an averaged brain (Desikan et al., 2006) was registered onto the brain of each individual, thus parcellating the white matter and cortex boundary of each brain into 68 regions of interest (supplemental Fig. S1, Table S1, available at www. jneurosci.org as supplemental material). The 3D T1-weighted anatomical images were registered and resampled onto the b0 of the diffusion scan using the rigid body registration tool of SPM8 (http://www.fil.ion.ucl.ac.uk/spm). The labeled white matter and cortex boundary that was originally defined in the T1-weighted scan space was then mapped onto the corresponding DTI scan space. These gyral-based cortical regions served as 68 nodes in the network of each individual.

Definition and computation of network edges. Network edges were defined as the interregional anatomical connections determined using DTI 
tractography. MedINRIA version 1.8.0 (http://www-sop.inria.fr/asclepios/ software/MedINRIA/index.php) was used to generate the white matter fiber bundles of the whole brain. The following default fiber-tracking settings of MedINRIA were used: FA threshold $=0.2$; smoothness $=20$; minimum length $=10 \mathrm{~mm}$; and sampling $=1$. An edge existed between two nodes (representing two cortical areas) if there was at least one fiber connecting them. Otherwise these two nodes were not directly connected (although they could still be connected via another node or nodes). Besides the route for each white matter fiber that had been generated, the number of fibers that connected every two cortical areas could also be estimated by initiating the same number of fibers in each voxel. We calculated the mean FA for each fiber, and then the average FA value of all the fibers that connected cortical areas $i$ and $j$ was used as the weighting $\left(w_{i j}\right)$ of the edge to describe the strength of the connectivity of cortical areas $i$ and $j$.

Using the binary information of whether or not any two nodes in the graph were directly connected and the quantitative information of the mean FA of the fibers if they were connected, we constructed two connectivity matrices for each subject, i.e., a binary matrix and a weighted matrix (see supplemental Figs. S3, S4, available at www.jneurosci.org as supplemental material). Each element of the binary matrix indicated whether the two cortical regions as labeled in the row and column were directly connected. In the weighted matrix, each element was the weighting factor for the two cortical areas. These two matrices determined the existence or absence and the weighting of an edge between two nodes that represented two regional cortical areas. An undirected, weighted graph was constructed for each subject (see supplemental Fig. S5, available at www.jneurosci.org as supplemental material) using these two matrices. As there were 68 cortical areas for each brain, the maximum number of all possible edges was $2278[N(N-1) / 2 ; N=68]$.

Sparsity of networks. The number of nodes was 68 , the same for all individual brain networks since each brain cortex was parcellated using the same scheme. However, the graph of each subject normally differs in both the number and weighting on the edges. The numbers of edges were likely to differ for different networks as the result of varying strengths and presence/absence of the connectivity of cortical areas in the subjects. Therefore, the corticocortical networks were examined using two different strategies. In the first strategy, the network of each subject was examined as it was constructed, i.e., all the edges were kept as long as there was a defined connection between two nodes. This was called the unmatched strategy as the number of edges of each individual network were different from each other. In the second strategy, which was called the matched strategy, a series of thresholds was set up on the connectivity matrices so that each graph would have the same number of edges under investigation. Computing and comparing graphs with the unmatched strategy is biologically meaningful as such graphs reflect the integrity and constraints of the brain networks they represent. On the other hand, both global and local network efficiencies have a propensity for being higher with greater numbers of edges in the graph. Therefore, besides the analyses on the networks with all the possible connections, i.e., using unmatched strategy, in the matched strategy, we also used the concept of sparsity to analyze the networks of not only the same number of nodes, but also the same number of edges, pertaining to the intrinsic network topological organization. For instance, if there were 228 edges in a graph of 68 nodes, the sparsity of the graph was 0.10 [228 divided by N(N$1) / 2 ; N=68$ ]. Obviously, altering sparsity values of a graph also altered the graph's structure. As a result of using a series of sparsity values for all individual brain networks, the interpretation of topological property comparisons were sometimes more meaningful and the individual brain network difference reflected the difference of the intrinsic properties of the graphs. Rather than setting a static sparsity value, we thresholded each connectivity matrix repeatedly over a wide range of sparsities, starting from the lowest at which the networks appeared to have small-world properties and finishing with the unthresholded networks. We then examined the graph topological attributes of the resulting graphs at each sparsity point.
Network small-worldness test

The "small-world" network, characterized by a high degree of clustering and short path length linking different network nodes, is an attractive model for the description of brain networks because it not only supports both specialized and integrated information processing but also minimizes wiring costs while maximizing the efficiency of information propagation (Watts and Strogatz, 1998; Kaiser and Hilgetag, 2006; Achard and Bullmore, 2007). A network is considered a small-world network if it has almost identical path length $\left(\lambda=L_{\mathrm{p}}^{\text {real }} / L_{\mathrm{p}}^{\text {rand }} \approx 1\right.$ ) (see Appendix for the mathematical expressions of brain network topology properties used in this work) but is more locally clustered $\left(\gamma=C_{\mathrm{p}}^{\text {real }} / C_{\mathrm{p}}^{\text {rand }}>1\right.$ and $\sigma=$ $\gamma / \lambda>1$ ) in comparison with the matched random networks (Watts and Strogatz, 1998). Therefore, to examine the small-world properties, the values of $L_{\mathrm{p}}^{\text {real }}$ and $C_{\mathrm{p}}^{\text {real }}$ of the weighted anatomical network were compared with $L_{\mathrm{p}}^{\text {rand }}$ and $C_{\mathrm{p}}^{\text {rand }}$, respectively. In this study, $L_{\mathrm{p}}^{\text {rand }}$ and $C_{\mathrm{p}}^{\text {rand }}$ were calculated from 2000 random networks generated using a previously determined random rewiring procedure (Maslov and Sneppen, 2002; Milo et al., 2002). These randomly generated networks had the same number of nodes and sparsities as the real networks constructed using each individual's DTI tractograph.

\section{Computation of network properties and statistical analysis}

The network properties computed included weighted global efficiency, weighted regional efficiency (as defined in Eqs. 16 and 18 in the Appendix, respectively), and their binarized counterparts, i.e., unweighted global efficiency and unweighted regional efficiency (as defined in Eqs. 15 and 17, respectively), using the Brain Connectivity Toolbox (http://sites. google.com/a/brain-connectivity-toolbox.net/bct/Home) (Rubinov and Sporns, 2009). The relevant mathematical definitions of network properties are listed in the Appendix.

All computations of network properties were performed by implementing algorithms using Matlab version R2008a (MathWorks). SPSS version 17 was then used for the statistical analyses. Linear regression analysis was used for the prediction of global network efficiency according to age and for the prediction of cognitive domain scores based on weighted global network efficiency. In all linear regression analyses, the sex and years of education of the participants were included in the models as control variables. Age was also included as a control variable, except for the regression analyses of global efficiency on age. In the analyses of age and cognitive functions versus regional connectivity efficiency of 68 cortical areas, partial correlation was used to control for age, sex, and years of education. Sex and years of education were controlled for in the age regional efficiency relationship analyses. The false discovery rate (FDR) (Benjamini and Hochberg, 1995) was used for multiplecomparison corrections.

\section{Results}

\section{Small-world properties of the DTI-tract-based brain networks}

The structural brain networks of all subjects had small-world architecture (unmatched strategy), i.e., network nodes had high levels of clustering and were linked through relatively few intermediate steps (supplemental Fig. S6, available at www.jneurosci. org as supplemental material) regardless of the age and cognitive performance of the participants. Although there were significant individual global and regional connectivity variations, the overall topological properties were largely invariant because smallworldness was preserved in all the structural networks studied.

\section{Age and cognitive functions versus global network efficiency} The networks that included all fibers present were examined, i.e., a connection (edge) between two cortical areas was considered to exist if there were one or more fibers connecting them. The weight of the edge was the mean FA value of the fibers that connected the two cortical areas. The number of edges in the 342 graphs ranged from 423 (sparsity: 0.185) to 863. Using a linear regression model, after controlling for sex and years of educa- 

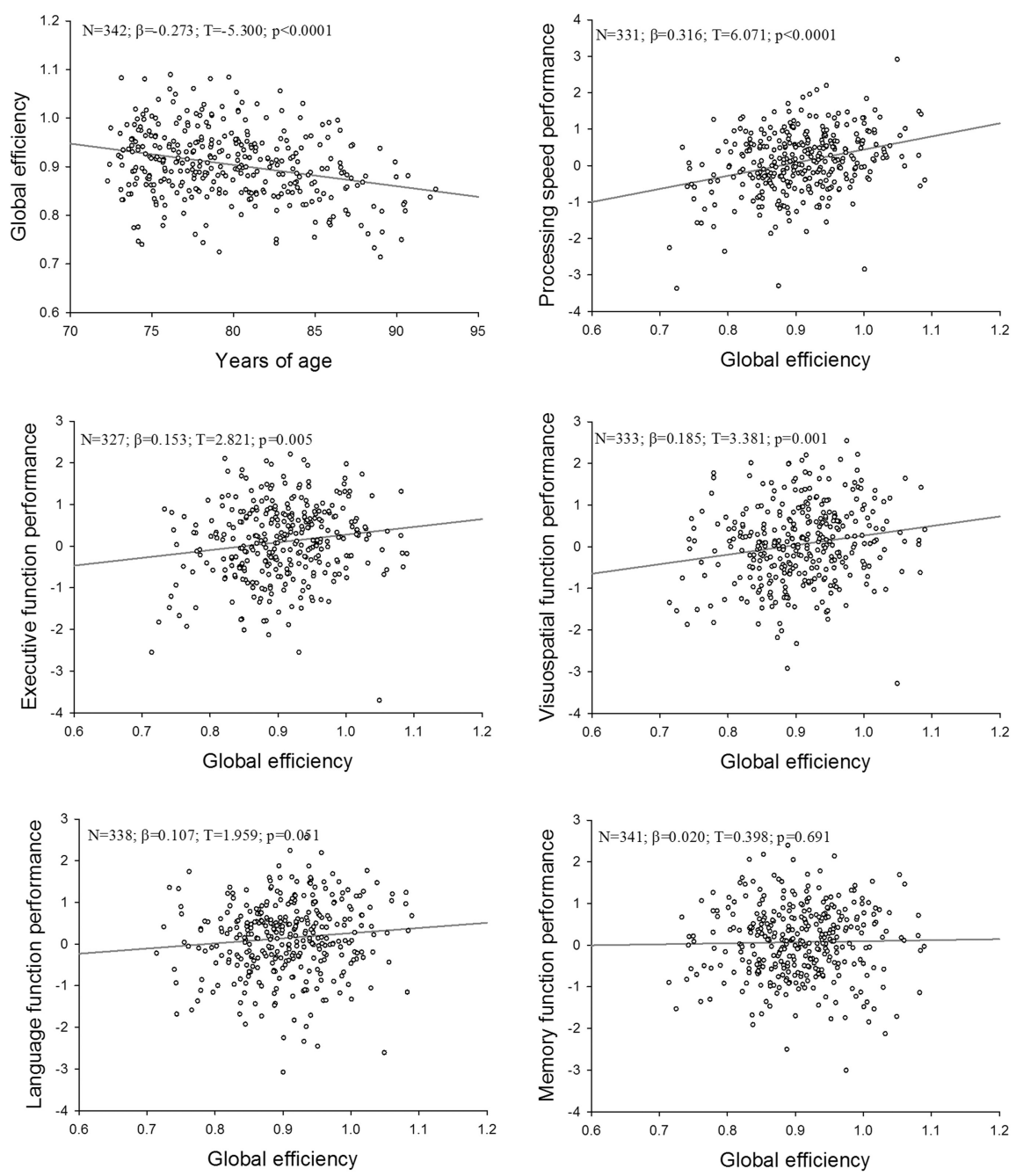

Figure 1. Regression analyses for the prediction of weighted global network efficiency from age, and for the prediction of cognitive domain scores from weighted global network efficiency. $N$, Number of subjects; $\beta$, standardized regression coefficient; $T, T$ value for regression coefficient; and $p$, significance of the regression coefficient. For the plot of age versus FA-weighted global efficiency, we used linear regression to remove the influence of sex and years of education from the weighted global efficiency values. For the other five plots, we used the cognitive scores (including processing speed, executive, visuospatial, language, and memory) that had been adjusted for age, sex, and years of education.

tion, we found that global network efficiency significantly reduced with older age $(N=342, \beta=-0.273, T=-5.300 ; p<$ $0.0001)$.

Higher weighted global network efficiency had a positive association with performances in processing speed $(N=331, \beta=$ $0.316, T=6.071 ; p<0.0001)$, visuospatial ability $(N=333, \beta=$ $0.185, T=3.381 ; p=0.001)$, and executive function $(N=327$, $\beta=0.153, T=2.821 ; p=0.005)$ after controlling for age, sex, and years of education. However, no significant association between global efficiency and memory performance $(N=341, \beta=$ $0.020, T=0.398 ; p=0.691)$ was found; and only marginal association between weighted global efficiency and language $(N=$ $338, \beta=0.107, T=1.959 ; p=0.051$ ) was found (Fig. 1). Some studies (Gong et al., 2009b; Yan et al., 2010) have considered brain size. Due to the fact that the variability in the number of edges in our 342 subjects was large, we performed a linear regression analysis before the analyses. Our linear regression analysis set the number of edges as the dependent variable and age, sex, ICV (intracranial volume), and total white matter volume as independent variables. We examined total white matter volume as well as ICV, as we consider that it is a better measure for studying white matter tracts. We found that neither $\operatorname{ICV}(N=$ $341, \beta=0.103, T=1.353 ; p=0.177)$ nor total white matter volume $(N=341, \beta=$ $0.120, T=1.664 ; p=0.097)$ significantly contributed to the number of edges. Conversely, age $(N=341, \beta=-0.189, T=$ $-3.448 ; p=0.001)$ and $\operatorname{sex}(N=341, \beta=$ $0.140, T=2.192 ; p=0.029)$ did have a significant correlation with number of edges. We therefore controlled for age, sex, and years of education.

Significant correlations were found between weighted global efficiency and processing speed, as well as visuospatial functions, at all sparsity values of $0.05-$ 0.18 (range of the number edges: 114-410) examined (see supplemental Table S2, available at www.jneurosci.org as supplemental material), providing further evidence for a robust association between these two cognitive functions and global efficiency of the weighted brain structural network.

\section{Age and cognitive functions versus regional network efficiency}

The regional efficiency of all 68 cortical regions (Fig. 2) was then examined. Age was found to have a negative effect association with regional efficiency of many cortical regions, including most of the frontal and temporal cortical areas and the entire cingulate cortex (59 regions out of 68 in total) (see Fig. 3 and supplemental Table S3, available at www.jneurosci.org as supplemental material). After controlling for age, sex, and years of education and correcting for multiple comparisons, higher regional connectivity efficiency of nearly all cortical regions was associated with greater processing speed (63 regions out of 68 in total) (see Fig. 3 and supplemental Table S4, available at www.jneurosci.org as supplemental material). Better performance in the visuospatial domain was related to higher regional connectivity efficiency of superior frontal gyrus of both left and right sides, some parts of middle frontal gyrus and inferior frontal gyrus, most cingulate cortex and insular cortex regions, and some left parietal regions (25 regions out of 68 in total) (see Fig. 3 and supplemental Table S5, available at www.jneurosci.org as supplemental material). Better executive performance was significantly correlated with greater regional connectivity of superior frontal gyrus of both left and right sides, posterior cingulate cortex of both left and right sides, left superior parietal cortex, left precuneus cortex, left caudal middle frontal gyrus, right precentral gyrus, right superior temporal gyrus, etc. (9 regions out of 68 in total) (see Fig. 3 and supplemental Table S6, available at www. jneurosci.org as supplemental material). Even though there were individual cortical regions that were associated with language, these correlations were not significant after corrections for multiple comparisons using FDR (see Fig. 3 and supplemental Table S7, available at www.jneurosci.org as supplemental material). 
There was no association between memory function and the connectivity of any of the 68 cortical regions. Since the memory domain examined consisted of several components, such as immediate and delayed narrative recall, short- and longterm delayed recall, and visual memory, we further analyzed the network connectivity in relation to each individual component. No significant correlation was found between the connectivity of any cortical region and these memory submeasures after multiple-comparison correction.

\section{Discussion}

The two main organizing principles found in the brain, i.e., segregation and integration of information processing (Friston, 2002; Sporns et al., 2005), provides a theoretical framework for conceptualizing the brain as a network. The approach of characterizing brain networks using connectivity matrices and graphs has the important advantage of a rich structural description that allows efficient computation and comparison of different connection topologies within a common theoretical framework (Bullmore and Sporns, 2009). Functional networks have previously been explored using functional MRI (fMRI) (Eguíluz et al., 2005; Salvador et al., 2005). Structural networks using interregional cortical thickness correlations (He et al., 2007) and DTI tractography (Hagmann et al., 2007; Iturria-Medina et al., 2008; Gong et al., 2009a) have been studied to understand the fundamental architecture of interregional connections. Some recent studies (Hagmann et al., 2008; Honey et al., 2009; Stephan et al., 2009) also reported a substantial correspondence between structural connectivity and functional connectivity. Using brain network approaches, attention has been paid to normal aging using fMRI (Achard and Bullmore, 2007), brain diseases such as AD (He et al., 2008) and multiple sclerosis (He et al., 2009) using interregional cortical thickness correlations, and schizophrenia using EEG (Rubinov et al., 2009), cortical volume correlations (Bassett et al., 2008), and fMRI (Liu et al., 2008).

Structural connectivity is best supported by fiber tracking, which demonstrates a physical connection between brain regions. DTI tractography provides this information and is ideal for such a connectivity study. Some recent analyses of the structural network using DTI (Hagmann et al., 2007, 2008; Iturria-Medina et al., 2008; Gong et al., 2009a) aimed to describe the network of elements and connections within the human brain, including the existence of modules, hubs, and structural cores. Age and sex (Gong et al., 2009b) and sex and brain size (Yan et al., 2010) have also been studied using DTI network approach.

Our study shows that the global efficiency of the corticocortical network decreases with age in the eighth and ninth decades of life. This decrease may be related to morphomolecular changes in neurons, axons, dendrites, and synapses, as well as the accumulation of neuropathologies that occur with age (Hof and Morri-

\section{Anterior}

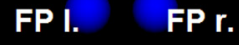

RMF I.

RAC I. MOF r.

RMF r.

PORB r.

LOF r.

PTRI r.

TP r.

POPE r.

CMF I.

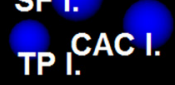

CAC r.

CMF r.

INS $r$.

ENT r.

ST r.

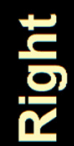

TT r. MT r.

TT I.

PC I. PC r.

PREC r.

IT I.

PARH I.

PARC I.

PARH r. PSTS r. IT r.

PARC r.

BSTS r.

BSTS I. FUS I.

ISTC I. ISTC $r$.

FUS r. SMAR r.

LING I. LING r.
I. SPI.
SP I. PCAL I.
PCAL $r$.
IP r.
LOCC I.
CUN I. CUN r.
SP r.
LOCC $r$.

PCUN I.

PCUN r.

\section{Posterior}

Figure 2. The abbreviations for the nodes of the network. Each node represents a cortical region, and there are 68 nodes in each network (34 in each hemisphere). The full names of the cortical regions are listed in supplemental Table 1 (available at www. jneurosci.org as supplemental material).

son, 2004). Nevertheless, one of the fundamentally important questions using a fiber-tracking-based network approach is how the network connectivity influences cognitive functions. This field has been largely unexplored except for one recent study that reported that general intelligence (Li et al., 2009) is associated with structural network properties such as network efficiency.

We examined for the first time not only the correlations of neuroanatomical connections as a system, but also whether neuroanatomical connectivity can be segregated to explain specific cognitive functions. The connections between segregated cortical regions may have two aspects. The first involves the existence or absence, and the strength and topological patterns, of the connections, i.e., the efficiency of the information exchange within the network. The second involves the effective coupling and grouping of certain cortical regions as neural sites that are required to respond together to different sensory inputs, task demands, and attentions. The disruption or weakening of the connections between certain cortical regions may therefore negatively affect some cognitive functions more than others. It is from this perspective that we should look into the regional efficiency of each individual cortical region. Cognitive functions depend not only on the patterns of network connectivity but also the coupling of cortical regions that global network efficiency may reflect. Processing speed is a reflection of an individual's ability to rapidly carry out many different types of processing 


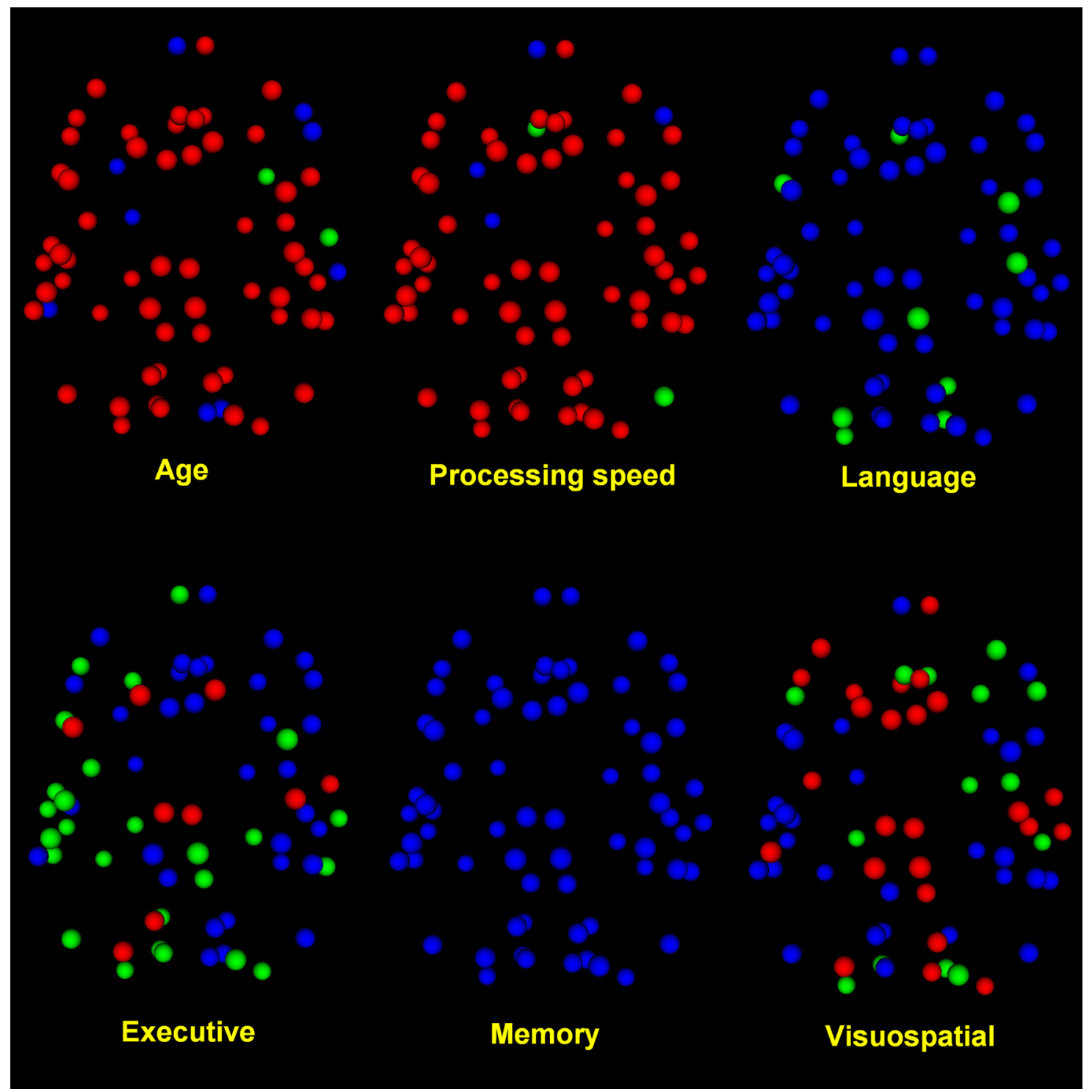

Figure 3. Age and cognitive functions versus regional efficiency for the FA-weighted networks of the 68 cortical areas (34 in each hemisphere). Partial correlation analyses were carried out. Age, sex, and years of education were controlled for in the cognitive function versus regional efficiency analyses (including processing speed, language, executive, memory, and visuospatial). In the age versus regional efficiency analyses, sex and years of education were controlled for. To correct for multiple comparisons, we used FDR to calculate the new thresholds for $p$ values, and $0.0320,0.0363$, 0.0190 , and 0.0025 were used for the multiple comparisons for age, processing speed, and visuospatial and executive functions, respectively. The colors of the nodes refer to: red, significant after FDR correction for multiple comparisons; green, $p<0.05$, significant before FDR correction; blue, $p \geq 0.05$, not significant.

operations, which are embedded in the particular task measured (Salthouse, 1996). It is therefore not surprising that poorer connectivity both globally in the brain and locally in large numbers of regional cortical areas could result in slower processing speed. The globally distributed nature of processing speed performance observed here is consistent with observations that brain lesions associated with variable etiologies and locations may commonly produce impairments in processing speed (DeLuca et al., 2004; Habekost and Rostrup, 2007; Willmott et al., 2009). Such a global network may be expected to be more vulnerable to disruptions due to age-related neuropathological changes in any part of the network resulting in a loss of efficiency and leading to a decline in processing speed that is typically associated with aging (Salthouse, 2000). Memory, however, may not be related to the same extent as processing speed to corticocortical connectivity because of the key role some subcortical structures such as the hippocampus play in this cognitive domain (Raz et al., 2005), and the connectivity between the hippocampus and other subcortical structures with cortical regions was not included in our study. Both language and memory assessments involve retrieval of lexical and semantic information and are supported by large-scale networks of anterior (frontal) and posterior (temporoparietal) language-related structures in both hemispheres of the brain with left-sided predominance. At the whole brain network level, there was some marginal association between weighted global efficiency and language. At the individual cortical regional level, some significant correlations were found, although these correlations became insignificant after multiple-comparison correction. In our study, we found that the regions that were significant (before correction for multiple comparisons) were consistent with the findings from studies looking at functional and structural correlates of these language tasks (Pihlajamäki et al., 2000; Grossman et al., 2004; Apostolova et al., 2008; Whitney et al., 2009). We could have expected to see significant findings in the 
left temporal region and medial temporal regions (hippocampus and parahippocampus) (Pihlajamäki et al., 2000; Whitney et al., 2009). One reason we did not observe stronger relationships between cortical networks and language could be that the tracts known to be implicated in language functions, namely the arcuate fasciculus and the inferior occipitofrontal fasciculus, which connect the anterior and posterior language areas, have an origin (or component) in subcortical regions, and in our study subcortical-cortical connections were not included. Visuospatial functions have been consistently associated with predominantly right posterior regions (Marshall and Fink, 2001). The test used to measure visuospatial function in this study, Block Design, places demands on higher-order cognitive functions, including perceptual reasoning and problem solving, in addition to more basic visuospatial perception (Lezak et al., 2004). Thus our finding of multiple local efficiency networks represented bilaterally, anteriorly, and posteriorly in the brain is consistent with the multifactorial nature of this test. Interestingly, the regional distribution of local networks associated with the executive domain was similar to the visuospatial domain, although fewer nodes survived statistical threshold after correction for multiple comparisons. Considerable controversy exists about precisely what is measured by tests of executive function. It has been suggested that tests used to assess executive functioning may not reflect a unique aspect of functioning but may be better explained in terms of other cognitive processes involved in test performance, such as verbal abilities, processing speed, or even intelligence (Greenaway et al., 2009; Roca et al., 2010). Hence, our executive domain may not be measuring a uniform set of processes, which may explain why only a small network was commonly expressed.

Regardless of the age and cognitive performance of the participants, we found that the brain structural networks of all subjects had small-world architecture, i.e., network nodes had high levels of clustering and were linked through relatively few intermediate steps. It is clear that although there were significant individual connectivity variations both globally and regionally, the overall topological properties were largely invariant as small-worldness was preserved in all of the structural networks that we studied.

Our study has some limitations. First, DTI tractography does not differentiate between efferent and afferent fibers, but we consider the FA of the fibers connecting two regions as a reasonable index of the strength of the neuronal traffic between them. Second, our neuroanatomical network consisted of only corticocortical connectivity information, due to our inability to automatically define the boundaries of subcortical structure with accuracy. An anatomical network including both cortical and subcortical regions may well be more informative of certain cognitive domains such as memory. Third, we used the mean FA values of the fibers as the weighting factor in the construction of the graphs, but other measures such as number of fibers or mean diffusivity, or a combination of measures, could also be considered as weighting factors. Number of fibers and average FA are measures of different aspects of the fibers, i.e., FA is relevant to fiber "integrity," and number of fibers is relevant to fiber "quantity." The process of quantifying diffusion indices and eventually comparing them between groups of subjects and/or correlating them with other parameters starts with the acquisition of the raw data, followed by a long series of image processing steps. A recent paper (Jones and Cercignani, 2010) points out that each of the processing steps is susceptible to sources of bias. Despite these limitations, our work demonstrates that the study of anatomical brain networks can inform us about age-related brain changes as well as the structural basis of cognitive functions.

\section{Appendix: Mathematical Expressions of Brain Network Topology Properties Used in This Work}

\section{Direct connection between two nodes}

For a binary graph $G$ with $N$ nodes, the connection status between any two nodes $(i, j)$ can be represented by $a_{i j}$, when an edge exists between these two nodes:

$$
a_{i j}=1
$$

otherwise:

$$
a_{i j}=0 \text {. }
$$

For a weighted graph $G^{w}$, the edge between nodes $(i, j)$ is given the weighting $w_{i j}$, which is a numerical value characterizing the strength of the connection.

\section{Degree of a node}

To perform an analysis of local property on the ith node, we define subgraph $G_{i}$, which includes all the directly connected neighboring nodes of the node $i$. One of the most fundamental network measures, with which most other measures are associated, is the degree of node $i$, denoted by $K_{i}$ for a binary graph and $K_{i}^{w}$ for a weighted graph (Bullmore and Sporns, 2009).

$$
K_{i}=\sum_{j \in G_{i}} a_{i j} ;
$$

and the weighted degree of node $i$ is as follows:

$$
K_{i}^{w}=\sum_{j \in G_{i}} w_{i j}
$$

\section{Clustering coefficient}

For an unweighted network, the clustering coefficient of node $i$ is the ratio of number of existing connections to the number of all possible connections in the subgraph $G_{i}$ :

$$
C_{i}=\frac{M_{G_{i}}}{K_{i}\left(1-K_{i}\right) / 2},
$$

where $M_{G_{i}}$ is the number of existing connections in $G_{i}$.

For a weighted network, we use the definition described by Onnela et al. (2005), in which the clustering coefficient is defined as follows:

$$
C_{i}^{w}=\frac{2}{K_{i}\left(K_{i}-1\right)} \sum_{j, m \in G_{i}}\left(W_{i j} W_{j m} W_{m i}\right)^{1 / 3},
$$

where $W_{i j}$ is the normalized weighting between a pair of nodes $i$ and $j$. Nodes $(j, m)$ are direct neighbors of node $i$, and these three nodes form a triangle. For weighted and unweighted graphs, we defined the averaged clustering coefficient respectively as

$$
C_{p}=\frac{1}{N} \sum_{i \in G} C_{i}
$$

and

$$
C_{p}^{w}=\frac{1}{N} \sum_{i \in G} C_{i}^{w}
$$




\section{Shortest path length}

The shortest path length $d_{i j}$ is the minimum number of edges that must be passed to go from one node to another in a binary network,

$$
d_{i j}=\sum_{a_{u v} \in P} a_{u v},
$$

where $P$ is the shortest path length between node $i$ and $j$.

However, for a weighted network, the shortest path length $d_{i j}^{w}$ is defined as the lowest-weighted path between node $i$ and $j$. In our study, a greater average FA value of all the fibers between two anatomical regions indicates stronger connection; thus, the connection that has a greater average FA values of all the fibers would have a shorter distance in the graph. Therefore, the weighting is the inverse of the average FA value. Note that these weightings are only used when computing the shortest path length:

$$
d_{i j}^{w}=\sum_{a_{u v} \in P^{w}} \frac{1}{w_{u v}} .
$$

The mean shortest path length of node $i$ is the average distance between node $i$ and all other nodes:

$$
L_{i}=\frac{1}{N} \sum_{\substack{i, j \in G \\ i \neq j}} d_{i j}
$$

and weighted:

$$
L_{i}^{w}=\frac{1}{N} \sum_{\substack{i, j \in G \\ i \neq j}} d_{i j}^{w}
$$

\section{Characteristic path length}

The characteristic path length (Watts and Strogatz, 1998) is the average mean shortest path length across all the nodes in the graph:

$$
L_{p}=\sum_{i \in G} L_{i}
$$

and the weighted graph:

$$
L_{p}^{w}=\sum_{i \in G} L_{i}^{w}
$$

\section{Global network efficiency}

Global efficiency is the inverse of the harmonic mean of the minimum absolute path length between each pair of nodes (Latora and Marchiori, 2001; Achard and Bullmore, 2007), for a binary graph:

$$
E_{\text {global }}=\frac{1}{N(N-1)} \sum_{j \in G} \sum_{i \in G, j \neq i} \frac{1}{d_{i j}} ;
$$

and the weighted graph:

$$
E_{\text {global }}^{w}=\frac{1}{N(N-1)} \sum_{j \in G} \sum_{i \in G, j \neq i} \frac{1}{d_{i j}^{w}} .
$$

\section{Regional network efficiency}

The regional efficiency is defined as the inverse of the mean harmonic shortest path length between the node under con- sideration and all other nodes in the network. For a binary graph:

$$
E_{i}=\frac{1}{N-1} \sum_{i \in G, j \neq i} \frac{1}{d_{i j}} ;
$$

and the weighted graph:

$$
E_{i}^{w}=\frac{1}{N-1} \sum_{i \in G, j \neq i} \frac{1}{d_{i j}^{w}} .
$$

\section{References}

Achard S, Bullmore E (2007) Efficiency and cost of economical brain functional networks. PLoS Comput Biol 3:e17.

Apostolova LG, Lu P, Rogers S, Dutton RA, Hayashi KM, Toga AW, Cummings JL, Thompson PM (2008) 3D mapping of language networks in clinical and pre-clinical Alzheimer's disease. Brain Lang 104:33-41.

Bassett DS, Bullmore E, Verchinski BA, Mattay VS, Weinberger DR, MeyerLindenberg A (2008) Hierarchical organization of human cortical networks in health and schizophrenia. J Neurosci 28:9239-9248.

Benjamini Y, Hochberg Y (1995) Controlling the false discovery rate-a practical and powerful approach to multiple testing. J R Stat Soc Series B Stat Methodol 57:289-300.

Benton AL, Spreen O (1996) Der Benton Test, Ed 7. Bern, Switzerland: Verlag Hand Huber.

Buckner RL (2004) Memory and executive function in aging and AD: multiple factors that cause decline and reserve factors that compensate. Neuron 44:195-208.

Bullmore E, Sporns O (2009) Complex brain networks: graph theoretical analysis of structural and functional systems. Nat Rev Neurosci 10:186-198.

Chen X, Wen W, Anstey KJ, Sachdev PS (2009) Prevalence, incidence, and risk factors of lacunar infarcts in a community sample. Neurology 73:266-272.

DeLuca J, Chelune GJ, Tulsky DS, Lengenfelder J, Chiaravalloti ND (2004) Is speed of processing or working memory the primary information processing deficit in multiple sclerosis? J Clin Exp Neuropsychol 26:550-562.

Desikan RS, Ségonne F, Fischl B, Quinn BT, Dickerson BC, Blacker D, Buckner RL, Dale AM, Maguire RP, Hyman BT, Albert MS, Killiany RJ (2006) An automated labeling system for subdividing the human cerebral cortex on MRI scans into gyral based regions of interest. Neuroimage 31:968-980.

Driscoll I, Hamilton DA, Petropoulos H, Yeo RA, Brooks WM, Baumgartner RN, Sutherland RJ (2003) The aging hippocampus: cognitive, biochemical and structural findings. Cereb Cortex 13:1344-1351.

Eguíluz VM, Chialvo DR, Cecchi GA, Baliki M, Apkarian AV (2005) Scalefree brain functional networks. Physical review letters 94:018102.

Fazekas F, Kleinert R, Offenbacher H, Schmidt R, Kleinert G, Payer F, Radner H, Lechner H (1993) Pathologic correlates of incidental MRI white matter signal hyperintensities. Neurology 43:1683-1689.

Friston K (2002) Beyond phrenology: what can neuroimaging tell us about distributed circuitry? Annu Rev Neurosci 25:221-250.

Gong G, He Y, Concha L, Lebel C, Gross DW, Evans AC, Beaulieu C (2009a) Mapping anatomical connectivity patterns of human cerebral cortex using in vivo diffusion tensor imaging tractography. Cereb Cortex 19:524-536.

Gong G, Rosa-Neto P, Carbonell F, Chen ZJ, He Y, Evans AC (2009b) Ageand gender-related differences in the cortical anatomical network. J Neurosci 29:15684-15693.

Greenaway MC, Smith GE, Tangalos EG, Geda YE, Ivnik RJ (2009) Mayo Older Americans Normative Studies: factor analysis of an expanded neuropsychological battery. Clin Neuropsychol 23:7-20.

Grossman M, McMillan C, Moore P, Ding L, Glosser G, Work M, Gee J (2004) What's in a name: voxel-based morphometric analyses of MRI and naming difficulty in Alzheimer's disease, frontotemporal dementia and corticobasal degeneration. Brain 127:628-649.

Gunning-Dixon FM, Raz N (2003) Neuroanatomical correlates of selected executive functions in middle-aged and older adults: a prospective MRI study. Neuropsychologia 41:1929-1941.

Habekost T, Rostrup E (2007) Visual attention capacity after right hemisphere lesions. Neuropsychologia 45:1474-1488. 
Hagmann P, Kurant M, Gigandet X, Thiran P, Wedeen VJ, Meuli R, Thiran JP (2007) Mapping human whole-brain structural networks with diffusion MRI. PLoS ONE 2:e597.

Hagmann P, Cammoun L, Gigandet X, Meuli R, Honey CJ, Wedeen VJ, Sporns O (2008) Mapping the structural core of human cerebral cortex. PLoS Biol 6:e159.

He Y, Chen ZJ, Evans AC (2007) Small-world anatomical networks in the human brain revealed by cortical thickness from MRI. Cereb Cortex 17:2407-2419.

He Y, Chen Z, Evans A (2008) Structural insights into aberrant topological patterns of large-scale cortical networks in Alzheimer's disease. J Neurosci 28:4756-4766.

He Y, Dagher A, Chen Z, Charil A, Zijdenbos A, Worsley K, Evans A (2009) Impaired small-world efficiency in structural cortical networks in multiple sclerosis associated with white matter lesion load. Brain 132:3366-3379.

Hof PR, Morrison JH (2004) The aging brain: morphomolecular senescence of cortical circuits. Trends Neurosci 27:607-613.

Honey CJ, Sporns O, Cammoun L, Gigandet X, Thiran JP, Meuli R, Hagmann P (2009) Predicting human resting-state functional connectivity from structural connectivity. Proc Natl Acad Sci U S A 106:2035-2040.

Iturria-Medina Y, Sotero RC, Canales-Rodríguez EJ, Alemán-Gómez Y, Melie-García L (2008) Studying the human brain anatomical network via diffusion-weighted MRI and graph theory. Neuroimage 40:1064-1076.

Jones DK, Cercignani M (2010) Twenty-five pitfalls in the analysis of diffusion MRI data. NMR Biomed 23:803-820.

Jorm AF, Butterworth P, Anstey KJ, Christensen H, Easteal S, Maller J, Mather KA, Turakulov RI, Wen W, Sachdev P (2004) Memory complaints in a community sample aged 60-64 years: associations with cognitive functioning, psychiatric symptoms, medical conditions, APOE genotype, hippocampus and amygdala volumes, and white-matter hyperintensities. Psychol Med 34:1495-1506.

Kaiser M, Hilgetag CC (2006) Nonoptimal component placement, but short processing paths, due to long-distance projections in neural systems. PLoS Comput Biol 2:e95.

Kaplan E, Goodglass H, Weintraub S (2001) Boston naming test, Ed 2. Philadelphia: Lippincott Williams \& Wilkins.

Kennedy KM, Raz N (2009) Aging white matter and cognition: differential effects of regional variations in diffusion properties on memory, executive functions, and speed. Neuropsychologia 47:916-927.

Latora V, Marchiori M (2001) Efficient behavior of small-world networks. Phys Rev Lett 87:198701.

Lezak M, Howieson D, Loring D (2004) Neuropsychological assessment. New York: Oxford UP.

Li Y, Liu Y, Li J, Qin W, Li K, Yu C, Jiang T (2009) Brain anatomical network and intelligence. PLoS Comput Biol 5:e1000395.

Liu Y, Liang M, Zhou Y, He Y, Hao Y, Song M, Yu C, Liu H, Liu Z, Jiang T (2008) Disrupted small-world networks in schizophrenia. Brain 131:945-961.

MacLeod CM (1991) Half a century of research on the Stroop effect: an integrative review. Psychol Bull 109:163-203.

Marshall JC, Fink GR (2001) Spatial cognition: where we were and where we are. Neuroimage 14:S2-7.

Maslov S, Sneppen K (2002) Specificity and stability in topology of protein networks. Science 296:910-913.

Milo R, Shen-Orr S, Itzkovitz S, Kashtan N, Chklovskii D, Alon U (2002) Network motifs: simple building blocks of complex networks. Science 298:824-827.

Morrison JH, Hof PR (2002) Selective vulnerability of corticocortical and hippocampal circuits in aging and Alzheimer's disease. Prog Brain Res 136:467-486.

Onnela JP, Saramäki J, Kertész J, Kaski K (2005) Intensity and coherence of motifs in weighted complex networks. Phys Rev E Stat Nonlin Soft Matter Phys 71:065103.

Pihlajamäki M, Tanila H, Hänninen T, Könönen M, Laakso M, Partanen K, Soininen H, Aronen HJ (2000) Verbal fluency activates the left medial temporal lobe: a functional magnetic resonance imaging study. Ann Neurol 47:470-476.

Prins ND, van Dijk EJ, den Heijer T, Vermeer SE, Jolles J, Koudstaal PJ, Hofman A, Breteler MM (2005) Cerebral small-vessel disease and decline in information processing speed, executive function and memory. Brain 128:2034-2041.

Raz N, Lindenberger U, Rodrigue KM, Kennedy KM, Head D, Williamson A, Dahle C, Gerstorf D, Acker JD (2005) Regional brain changes in aging healthy adults: general trends, individual differences and modifiers. Cereb Cortex 15:1676-1689.

Reitan RM, Wolfson D (1985) The Halstead-Reitan neuropsychological test battery: theory and clinical interpretation. Tucson, AZ: Neuropsychology Press.

Rey A (1964) L'Examen Clinique en Psychologie. Paris: Presses Universitaires de France.

Roca M, Parr A, Thompson R, Woolgar A, Torralva T, Antoun N, Manes F, Duncan J (2010) Executive function and fluid intelligence after frontal lobe lesions. Brain 133:234-247.

Rubinov M, Sporns O (2010) Complex network measures of brain connectivity: uses and interpretations. Neuroimage 52:1059-1069.

Rubinov M, Knock SA, Stam CJ, Micheloyannis S, Harris AW, Williams LM, Breakspear M (2009) Small-world properties of nonlinear brain activity in schizophrenia. Hum Brain Mapp 30:403-416.

Salat DH, Kaye JA, Janowsky JS (2002) Greater orbital prefrontal volume selectively predicts worse working memory performance in older adults. Cereb Cortex 12:494-505.

Salthouse TA (1996) The processing-speed theory of adult age differences in cognition. Psychol Rev 103:403-428.

Salthouse TA (2000) Aging and measures of processing speed. Biol Psychol 54:35-54.

Salvador R, Suckling J, Coleman MR, Pickard JD, Menon D, Bullmore E (2005) Neurophysiological architecture of functional magnetic resonance images of human brain. Cereb Cortex 15:1332-1342.

Smith SM, Jenkinson M, Woolrich MW, Beckmann CF, Behrens TE, Johansen-Berg H, Bannister PR, De Luca M, Drobnjak I, Flitney DE, Niazy RK, Saunders J, Vickers J, Zhang Y, De Stefano N, Brady JM, Matthews PM (2004) Advances in functional and structural MR image analysis and implementation as FSL. Neuroimage 23 [Suppl 1]:S208-S219.

Sporns O, Tononi G, Kötter R (2005) The human connectome: a structural description of the human brain. PLoS Comput Biol 1:e42.

Spreen O, Benton AL (1969) Neurosensory Center Comprehensive Examination for Aphasia: manual of instructions (NCCEA). Victoria, BC: University of Victoria.

Stephan KE, Tittgemeyer M, Knösche TR, Moran RJ, Friston KJ (2009) Tractography-based priors for dynamic causal models. Neuroimage 47:1628-1638.

Strauss E, Sherman EMS, Spreen O (2006) A compendium of neuropsychological tests: administration, norms, and commentary, Ed 3. New York: Oxford UP.

Watts DJ, Strogatz SH (1998) Collective dynamics of 'small-world' networks. Nature 393:440-442.

Wechsler D (1981) WAIS-R manual. New York: The Psychological Corporation.

Wechsler D (1997) Wechsler adult intelligence scale-III. San Antonio: The Psychological Corporation.

Wen W, Sachdev PS (2004) Extent and distribution of white matter hyperintensities in stroke patients: the Sydney Stroke Study. Stroke 35:2813-2819.

Whitney C, Weis S, Krings T, Huber W, Grossman M, Kircher T (2009) Task-dependent modulations of prefrontal and hippocampal activity during intrinsic word production. J Cogn Neurosci 21:697-712.

Willmott C, Ponsford J, Hocking C, Schönberger M (2009) Factors contributing to attentional impairments after traumatic brain injury. Neuropsychology 23:424-432.

Yan C, Gong G, Wang J, Wang D, Liu D, Zhu C, Chen ZJ, Evans A, Zang Y, He Y (2010) Sex- and brain size-related small-world structural cortical networks in young adults: a DTI tractography study. Cereb Cortex. Advance online publication. Retrieved Dec. 19, 2010. doi:10.1093/cercor/bhq111. 